\title{
Targeted and Efficient Delivery of siRNA Using Tunable Polymeric Hybrid Micelles for Tumor Therapy
}

\author{
FEI HAO ${ }^{1}$, SHIYAN DONG ${ }^{1}$, CHUNMIAO YANG ${ }^{1}$, ZIWEI LI ${ }^{1}$, ZIYUAN CHENG ${ }^{1}$, \\ LIHUANG ZHONG ${ }^{1}$, LIRONG TENG ${ }^{1}$, QINGFAN MENG ${ }^{1}$, JIAHUI LU ${ }^{1}$, \\ FAN WU ${ }^{2}$, JING XIE ${ }^{1}$, LESHENG TENG $^{1}$ and ROBERT J. LEE ${ }^{1,3}$ \\ ${ }^{1}$ School of Life Sciences, Jilin University, Changchun, P.R. China; \\ ${ }^{2}$ College of Medicine, Des Moines University, Des Moines, IA, U.S.A.; \\ ${ }^{3}$ College of Pharmacy, The Ohio State University, Columbus, OH, U.S.A.
}

\begin{abstract}
Background/Aim: Effective and targeted delivery of siRNA to tumor cells is a prerequisite to achieving their therapeutic effects. Survivin is up-regulated in tumor cells and is associated with resistance to therapy. Therefore, siRNAmediated silencing of survivin is a potential therapeutic strategy for cancer. The aim of the study was to examine whether polymeric hybrid micelles can be used to effectively deliver siRNAs into cells. Materials and Methods: First, linoleic acid (LA) was conjugated to polyethylenimine (PEI) and methoxy-polyethyleneglycol ( $m P E G)$ and two amphiphilic polymers (PEI-LA and mPEG-LA) were obtained. Polymeric hybrid micelle (PHM) was then prepared and characterized by self-assembly of PEI-LA and mPEG-LA at different percentages of the two amphiphilic polymers. A PHM/siRNA complex with optimized composition and good biocompatibility was then prepared and its cellular uptake, biodistribution, and antitumor effects were investigated. Results: Survivin siRNA was efficiently delivered to the cells. It reduced survivin protein expression and greatly suppressed tumor growth. Moreover, siRNA loaded in PHM gathered in a solid tumor in mice and achieved an improved anticancer effect compared to naked siRNA. Conclusion: PHM is a promising and safe vehicle for siRNA delivery and may find utility in cancer therapy.
\end{abstract}

This article is freely accessible online.

Correspondence to: Robert J. Lee, College of Pharmacy, The Ohio State University, Columbus, OH 43210, USA. Tel: +1 6142924172, e-mail: lee.1339@osu.edu; or Xie Jing, School of Life Sciences, Jilin University, No.2699, Qianjin Street, Changchun 130012, PR China. Tel: +(86) 13843004264, e-mail: xiejing@jlu.edu.cn; or Lesheng Teng, School of Life Sciences, Jilin University, No.2699, Qianjin Street, Changchun 130012, PR China. Tel: +(86) 13844181693,e-mail: tenglesheng@jlu.edu.cn

Key Words: Polymeric hybrid micelle, siRNA, targeted delivery, anticancer effect.
Small interfering RNA (siRNA) with a length of 20-25 base pairs can specifically silence the expression of a targeted gene through RNA interference $(1,2)$. siRNA has potential application in the treatment of tumors (3). However, it still remains a challenge to efficiently deliver siRNA to target tissues (4). Therefore, a safe and effective delivery system designed for siRNA is essential.

Non-viral vectors are widely used for siRNA delivery (57). Branched polyethylenimine (PEI), as a cationic polymer, has been used in cellular transfections $(8,9)$ as it has the ability to form complexes with siRNA, protect it from degradation, enhance interaction with cell membranes, increase cellular uptake and help siRNA to escape the endosomal pathway due to its proton sponge effect $(5,6,8$, 10-13). However, PEI application is limited due to its high toxicity $(14,15)$. Current research has focused on the modification of PEI by hydrophobic or hydrophilic moieties to reduce its toxicity (15-19). Studies have shown that the modification of PEI with hydrophobic fatty acids such as oleic acid, stearic acid, and linoleic acid could significantly reduce the toxicity of PEI and increase its transfection efficiency in vitro (20-22). However, its application in vivo is still limited.

Polyethyleneglycol (PEG) coating is often used to shield the charge, reduce the toxicity, increase the biocompatibility, and prolong the circulation time of the cationic carrier in the blood (23-27). It has been directly conjugated to PEI and formed copolymers used for siRNA delivery $(26,28)$. However, PEG may interfere with the interaction of PEI with the siRNA $(11,24)$. Polymeric hybrid micelle (PHM) composed of two or more amphiphilic block copolymers are emerging as a promising delivery system with high flexibility (29-31). The properties of PHM that are important for siRNA delivery can be tuned by simply adjusting the proportion of the cationic carrier or PEG-based block polymer (11, 29, 32, 33). In this study, linoleic acid (LA)-modified mPEG and PEI were synthesized. Survivin is an anti-apoptotic factor that is upregulated in tumor cells and is associated with therapy 
resistance (34). Therefore, siRNA-mediated silencing of survivin is a potential therapeutic strategy for cancer. Survivin siRNA was loaded in PHM. The cellular uptake and biological activity in vitro, the biodistribution and the anti-tumor effect of survivin siRNA in vivo were evaluated.

\section{Materials and Methods}

Materials. Branched polyethylenimine (PEI, $25 \mathrm{kDa}$ ) was purchased from Sigma-Aldrich (St. Louis, MO, USA). 3-(4,5-DEimethyl-2thiazolyl)-2,5-diphenyl-2-H-tetrazolium bromide (MTT) was purchased from Shanghai Yuanye Biological Technology (Shanghai, China). mPEG-NH $\mathrm{NH}_{2}$ (2,000Da) was purchased from Yarebio (Shanghai, China). Linolyl chloride (LC) was obtained from Tokyo Chemical Industry Co., Ltd. (Shanghai, China). Survivin siRNA: Sense (5'-3'): ' GCAGGUUCCUmUAUCUGUCAdTdT; Antisense (5'-3'): UGA ${ }^{\mathrm{m}} \mathrm{CAGA}^{\mathrm{m} U A A G G A A C C U G}{ }^{\mathrm{m}} \mathrm{C}$ dTdT and fluorescencelabeled survivin siRNA that had $\mathrm{Cy} 3$ or $\mathrm{Cy} 5$ conjugated to the 5' end for cellular uptake and bio-distribution studies were synthesized by Ribo Biochemistry (Guangzhou, China). A549 cells were purchased from ATCC (Rockefeller, MD, USA). All chemical reagents used were of analytical grade.

Synthesis and characterization of the synthesized amphiphilic polymers. LA was separately conjugated to the mPEG and PEI using a previously published method (20-22). Briefly, LC dissolved in anhydrous dichloromethane (DCM) (Sinopharm Chemical Reagent Co., Ltd., Shanghai, PR China) was added dropwise to the $\mathrm{mPEG}_{2000}-\mathrm{NH}_{2}$ and $\mathrm{PEI}_{25 \mathrm{kDa}}$ anhydrous DCM solution, respectively. After $12 \mathrm{~h}$, the reaction mixture was precipitated and washed three times by diethyl ether. The products mPEG-LA and bPEI-LA were obtained by removing diethyl ether by a rotary evaporator (Shanghai Yukang Scientific Instrument Co., Ltd., Shanghai, PR China) and vacuum drying.

Preparation of polymeric hybrid micelle (PHM). PHM was prepared by self-assembly of PEI-LA and mPEG-LA. Different weight ratios PEI-LA and mPEG-LA were mixed together in DCM and sonicated. Then, the mixture solution was evaporated by a rotary evaporator to remove the organic solvent and further vacuumed for $2 \mathrm{~h}$ to remove residual organic solvents. To prepare PHM, diethyl pyrocarbonate (DEPC)-treated water (Coolaber, Beijing, PR China) was added to the flasks and sonicated for $2 \mathrm{~min}$. Particle size, zeta potential, and polydispersity index (PDI) of PHM were measured on a Zeta-sizer Nano ZS90 from Malvern Instruments (Malvern, UK) at $25^{\circ} \mathrm{C}$.

Hemolytic analysis of PHM. Fresh blood samples from healthy mice were collected from the orbital sinus in heparin-coated tubes. Red blood cells (RBCs) were collected by centrifuging at $3000 \mathrm{rpm}$ for 5 min and washed three times with physiological saline solution. Then, the RBCs were dispersed in the physiological saline solution to obtain a $2 \%$ RBCs standard dispersion (v/v). PHM (100 $\mu \mathrm{l}, 5 \mathrm{mg} / \mathrm{ml})$ was incubated with $0.5 \mathrm{ml} \mathrm{RBCs}$ standard dispersion for $3 \mathrm{~h}$. The suspensions were centrifuged, and the absorbance of supernatant was measured at $450 \mathrm{~nm}$.

Gel retardation assay. Gel retardation assay was conducted to study the ability of PHM to complex siRNA using agarose gel electrophoresis. PHM was first incubated with survivin siRNA for
$10 \mathrm{~min}$ at different PEI nitrogen-to-RNA phosphate (N/P) ratios. Then, $10 \mu \mathrm{l}$ of the PHM/siRNA complexes with different N/P ratios were loaded onto an agarose gel $(2 \%, \mathrm{w} / \mathrm{v})$. The electrophoresis was set up at $100 \mathrm{~V}$ and run for $10 \mathrm{~min}$ in a Tris-acetate-EDTA (TAE) buffer (Beijing Dingguo Changsheng Biotechnology Co., Ltd., Beijing, PR China). The gel was stained with ethidium bromide (Beijing Dingguo Changsheng Biotechnology Co., Ltd., Beijing, PR China) and imaged by a UV transilluminator (Analytik Jena US LLC., Upland, CA, USA).

Cellular uptake of the PHM/Cy3-siRNA. A549 cells were cultured in Dulbecco's modified eagle medium (DMEM) (Invitrogen Co., Carlsbad, CA, USA) which contained $10 \%$ fetal bovine serum (FBS) (Gemini, Woodland, CA, USA) and $1 \%$ penicillinstreptomycin (Carlsbad, CA, USA) at $37^{\circ} \mathrm{C}$ in a humidified atmosphere of $5 \% \mathrm{CO}_{2}$. A549 cells were seeded in 12-well cell culture plates with $1 \times 10^{5}$ cells/well and cultured for $24 \mathrm{~h}$. Naked Cy3-siRNA and PHM/Cy3-siRNA with an equivalent siRNA concentration of $100 \mathrm{nM}$ were then added to the wells and incubated with the cells for $4 \mathrm{~h}$. A549 cells were then trypsinized, harvested and fixed with $4 \%(\mathrm{w} / \mathrm{v})$ cold formaldehyde solution (Beijing Dingguo Changsheng Biotechnology Co., Ltd., Beijing, PR China). The fluorescence intensity of the cells was measured on a Beckman Coulter EPICS XL flow cytometer (Brea, CA, USA).

The cellular uptake of PHM/Cy3-siRNA was further imaged by confocal laser scanning microscopy (CLSM). A549 cells were seeded at the bottom of a glass flask and cultured for $12 \mathrm{~h}$. The cells were then treated with naked siRNA or PHM/Cy3-siRNA $(100 \mathrm{nM})$ at $37^{\circ} \mathrm{C}$. After $4 \mathrm{~h}$ of incubation, the medium was removed and the cells were washed with phosphate buffer solution (PBS, 0.01 M, pH 7.4). Then, the cells were fixed with $4 \%(\mathrm{w} / \mathrm{v})$ formaldehyde for $15 \mathrm{~min}$ at room temperature and stained with 4',6-Diamidino-2-phenylindole (DAPI) (Invitrogen Co., Carlsbad, CA, USA) for $15 \mathrm{~min}$. Uptake of the PHM/Cy3-siRNA complexes from A549 cells was studied using an LSM710 microscope from Carl Zeiss (Oberkochen, Germany).

Cytotoxicity of the PHM/survivin-siRNA. A549 cells were plated in 96-well microtiter plates ( 8000 cells per well) and cultured overnight. Naked survivin-siRNA, PHM and PHM/survivin-siRNA were added to the wells (survivin siRNA $40 \mathrm{nM}, 80 \mathrm{nM}, 100 \mathrm{nM}$ ). After cultured for $48 \mathrm{~h}, 20 \mu \mathrm{l}$ of MTT solution $(5 \mathrm{mg} / \mathrm{ml})$ were added to the well and incubated with the cells for $4 \mathrm{~h}$. The medium was removed and $150 \mu \mathrm{l}$ DMSO was added. Absorbance values at $490 \mathrm{~nm}$ were measured on Bio Tek SYNERGY4 (Winooski, VT, USA). Relative cell viability was presented as a percentage of viability treated with different formulations compared to untreated cell samples.

Western blot assay. A549 cells were seeded in 6-well cell culture plates $\left(1 \times 10^{5}\right.$ cells /per well) for $24 \mathrm{~h}$ at $37^{\circ} \mathrm{C}$ in $5 \% \mathrm{CO}_{2}$ humidified atmosphere. Naked survivin-siRNA and PHM/survivin-siRNA were added to the wells (survivin siRNA $80 \mathrm{nM}$ ). At $48 \mathrm{~h}$ later, cells were collected and lysed in radio immunoprecipitation assay (RIPA) lysis buffer (Sigma-Aldrich, St. Louis, MO, USA). Protein fractions were collected, quantified, subjected to polyacrylamide gel electrophoresis, and finally transferred to polyvinylidene fluoride (PVDF) membrane (0.45 um, Merck Millipore, Billerica, MA, USA). The membrane was blocked with 5\% BSA for $4 \mathrm{~h}$ at room temperature and incubated with survivin Rabbit mAb (71G4B7E, Cell Signaling Technology Inc, Danvers, MA, USA) and GAPDH Rabbit antibodies (Beijing Dingguo Changsheng Biotechnology Co., 


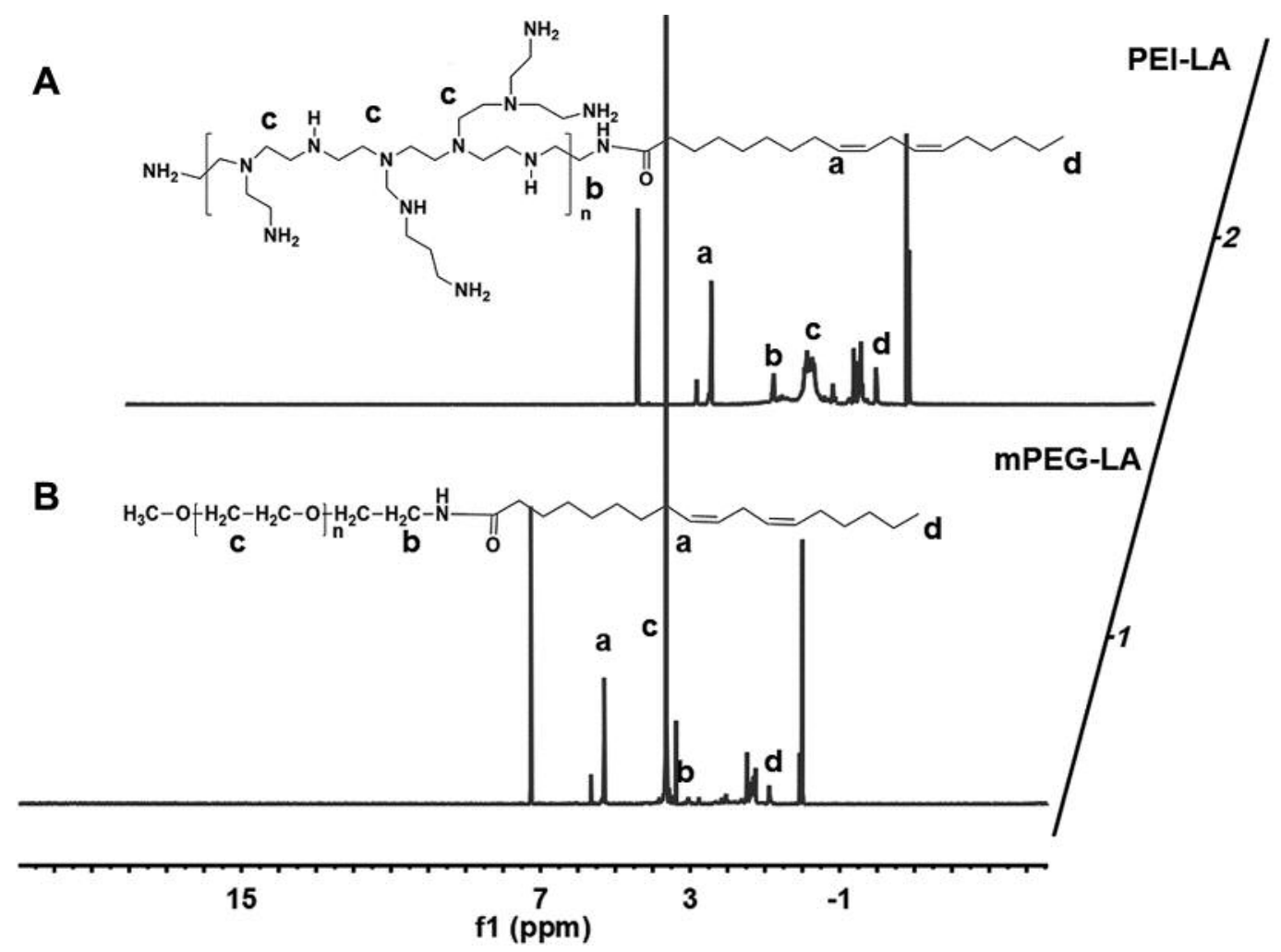

Figure 1. The ${ }^{1} H$ NMR of PEI-LA and mPEG-LA. PEI-LA and mPEG-LA were dissolved in $C^{2} C_{3}$ and measured in a nuclear magnetic resonance instrument (400 MHZ). The characteristic peaks of PEI-LA and mPEG-LA were marked with the characters $(a, b, c, d)$.

Ltd., Beijing, PR China) at $4^{\circ} \mathrm{C}$ overnight. Horseradish peroxidase (HRP)-conjugated secondary antibody (Beijing Dingguo Changsheng Biotechnology Co., Ltd., Beijing, PR China) was then added and incubated at $4^{\circ} \mathrm{C}$ for $4 \mathrm{~h}$. The corresponding protein expression was visualized by an imaging system (BioSpectrum600, Analytik Jena US LLC., Upland, CA, USA).

Establishment of a tumor model. The animal experimental protocol was complied with the institutional guidelines and was approved by the Experimental Animal Ethics Committee of the School of Life Sciences, Jilin University (approval number 201805003). BALB/c nude mice (female, 6-8 weeks) were obtained from Beijing Vital River Laboratory Animal Technology Co., Ltd. (Beijing, PR China). Tumor-bearing mice were established through subcutaneous injection of $5 \times 10^{6} \mathrm{~A} 549$ cells into the right rear leg of nude mice after the mice were adapted to the new environment for a week.

Biodistribution of PHM/Cy5-siRNA complexes in tumor-bearing mice. Tumor-bearing mice were injected with Cy5-siRNA and PHM/Cy5-siRNA via the tail vein with an equivalent amount of Cy5-siRNA $(0.8 \mathrm{nmol})$. Mice were anesthetized and the biodistribution of the complexes was imaged on an IVIS ${ }^{\circledR}$ spectrum system from Caliper Life Sciences (Hopkinton, MA, USA) at the first, second, and fourth $\mathrm{h}$ after administration with an optimized parameter (Excitation at $640 \mathrm{~nm}$ and Emission at $680 \mathrm{~nm}$ ). After $6 \mathrm{~h}$, internal organs (Heart, Liver, Spleen, Lung, and Kidney) and tumors were dissected and then imaged.
Therapeutic efficacy of $\mathrm{PHM} /$ siRNA in vivo. When the average tumor volume of nude mice was grown to approximately $100-150 \mathrm{~mm}^{3}$ (Day 0), animals were randomized into three groups, each containing 5 nude mice. Tumor-bearing mice were then injected with saline, free siRNA and PHM/siRNA via intravenous injection every 3 days. The dose of naked siRNA and PHM/siRNA was 4 nmol per mice. The volume of the tumor and body weight were measured every 3 days using a vernier caliper and scale. On day 20 , mice were anesthetized with sodium pentobarbital and sacrificed. Vital organs (Heart, Liver, Spleen, Lung, and Kidney) and tumors were dissected in each group and fixed with $4 \%$ paraformaldehyde. Tissue sections were cut into 5 microns thick and stained with hematoxylin and eosin (H\&E). The expression of survivin in tumor tissue was analyzed by western blot assay.

Statistical analysis. Data were presented as mean \pm SEM and graphed by Origin 8.0 (OriginLab Corp., Northampton, MA, USA). Statistical analysis of group differences and correlations was determined using Student's $t$-test. $* p<0.05$ was considered statistically significant.

\section{Results}

Synthesis and characterization of PEI-LA and MPEG-LA. The chemical structures of PEI-LA and mPEG-LA were confirmed by ${ }^{1} \mathrm{H}$ NMR (Figure $1 \mathrm{~A}$ and $\mathrm{B}$ ). In Figure 1A, the characteristic peak assignments of LA $(a, \delta=5.34$ ppm for 1- 

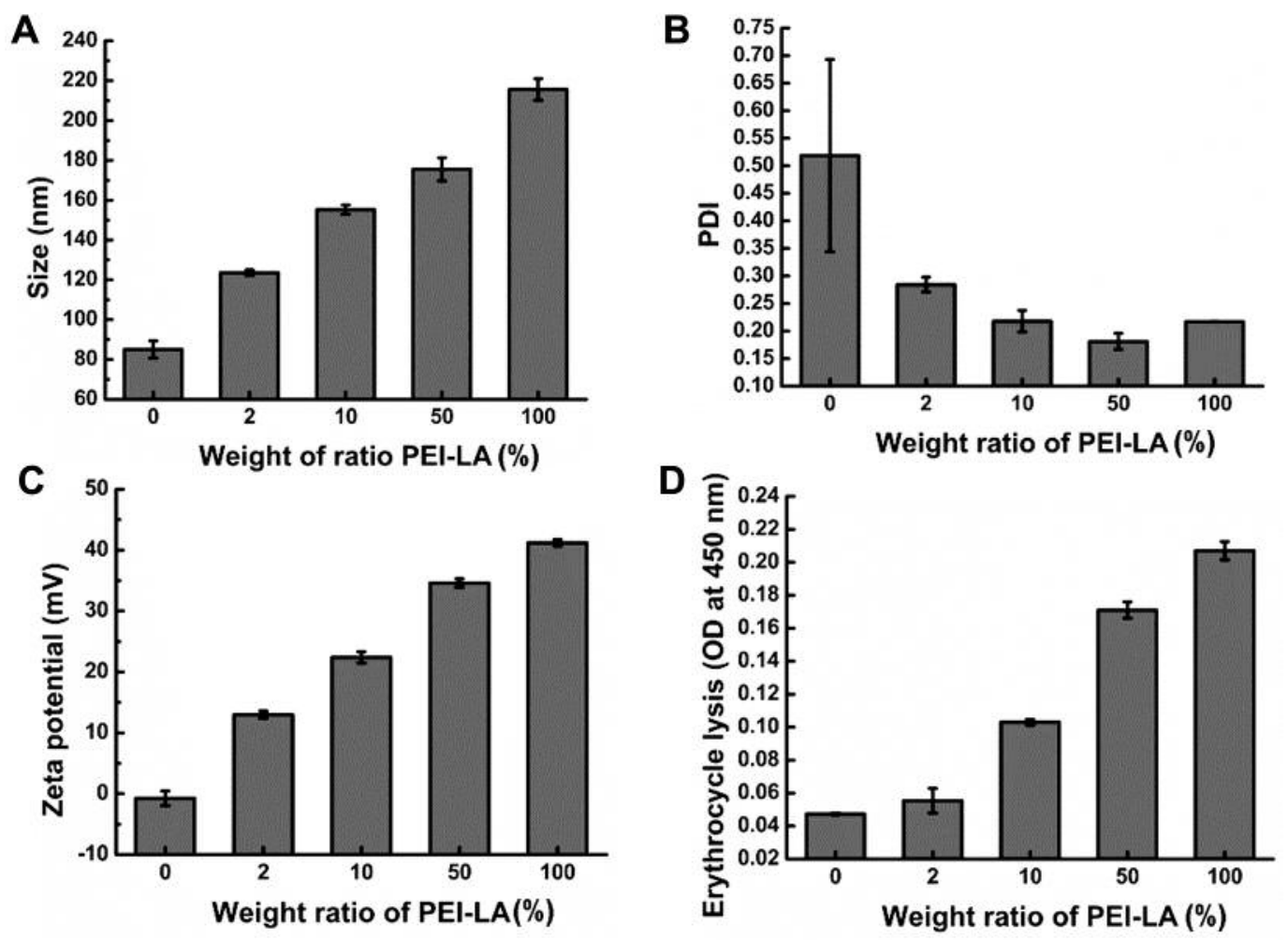

Figure 2. Characterization of PHM at different \% of PEI-LA. Particle size (A), PDI (B) and zeta potential (C) of PHM was measured on a Zetasizer Nano ZS90 (n=3, mean \pm SEM). (D)The hemolytic activity of PHM on mice erythrocytes with a different\% of PEI-LA $(n=3$, mean \pm SEM $)$.
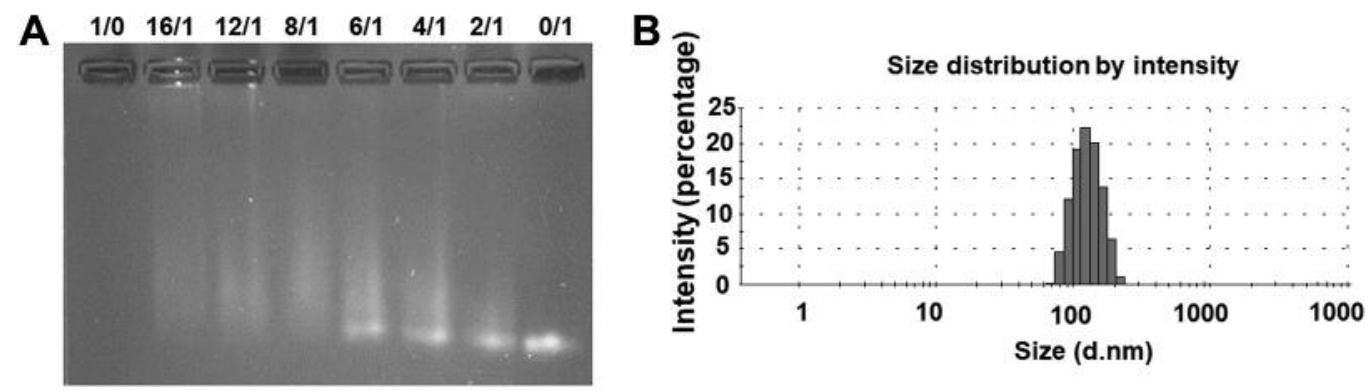

Figure 3. Characterization of PHM/siRNA complexes. (A) Gel retardation assay of PHM/siRNA complexes with different N/P ratio. (B) The particle size of the PHM/siRNA with an N/P ratio of 12/1.

ethylene; $\mathrm{d}, \delta=0.88 \mathrm{ppm}$ for methyl) and PEI (c, $\delta=2.3-3.00$ ppm) are marked out. The new characteristic peak assignment of methylene in $1 \alpha-\mathrm{N}(\mathrm{C})-\mathrm{C}(\mathrm{b}, \delta=3.63 \mathrm{ppm})$ indicated amide bond generation and that LA was successfully linked to the PEI. As shown in Figure 1B, the characteristic peaks for 1-ethylene ( $\mathrm{a}, \delta=5.34 \mathrm{ppm})$, methyl $(\mathrm{d}, \delta=0.86 \mathrm{ppm})$ of LA were also further confirmed in $1 \mathrm{H}$ NMR of mPEG-LA. The characteristic peak assignment of methylene in PEG was marked with c $(\delta=3.64 \mathrm{ppm})$.
Similarly, the new characteristic peak assignment of methylene in $1 \alpha-\mathrm{NC}=\mathrm{O}-\mathrm{C}$ (b) due to the new amide bond generation also appeared at $3.37 \mathrm{ppm}$. The peak assignment of the methylene in $1 \alpha-\mathrm{NC}=\mathrm{O}-\mathrm{C}$ indicated that the $\mathrm{LC}$ was also successfully conjugated to mPEG-NH2. The purity of the mPEG-LA and PEI-LA was calculated to be $91 \%$ and $94.7 \%$ based on the characteristic peak integration. And then the degree of functionalization of PEI with LA moieties was calculated to be 12 . 
A

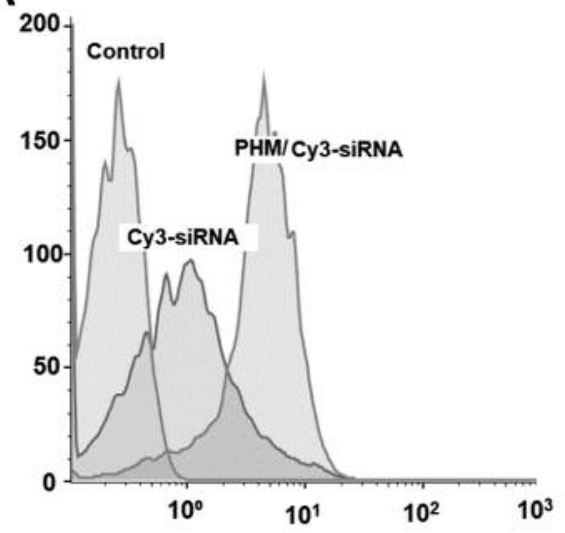

B

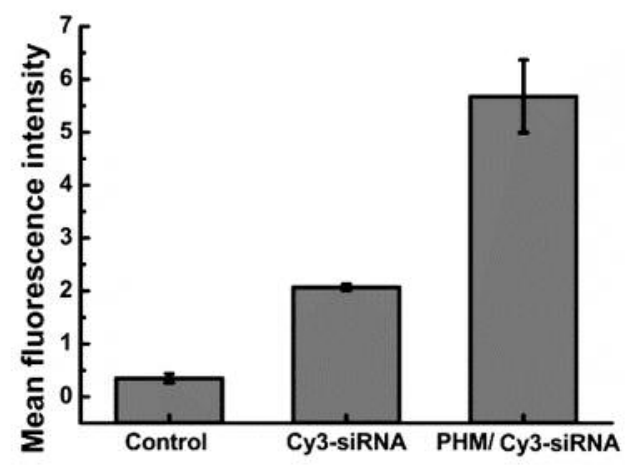

Figure 4. Cellular uptake of PHM/Cy3-siRNA by flow cytometry. Cellular uptake of naked Cy3-siRNA and PHM/ Cy3-siRNA in the A549 cells (A) and the mean fluorescence intensity was further quantified $(B)(n=3$, mean $\pm S E M)$ at $4 \mathrm{~h}$.

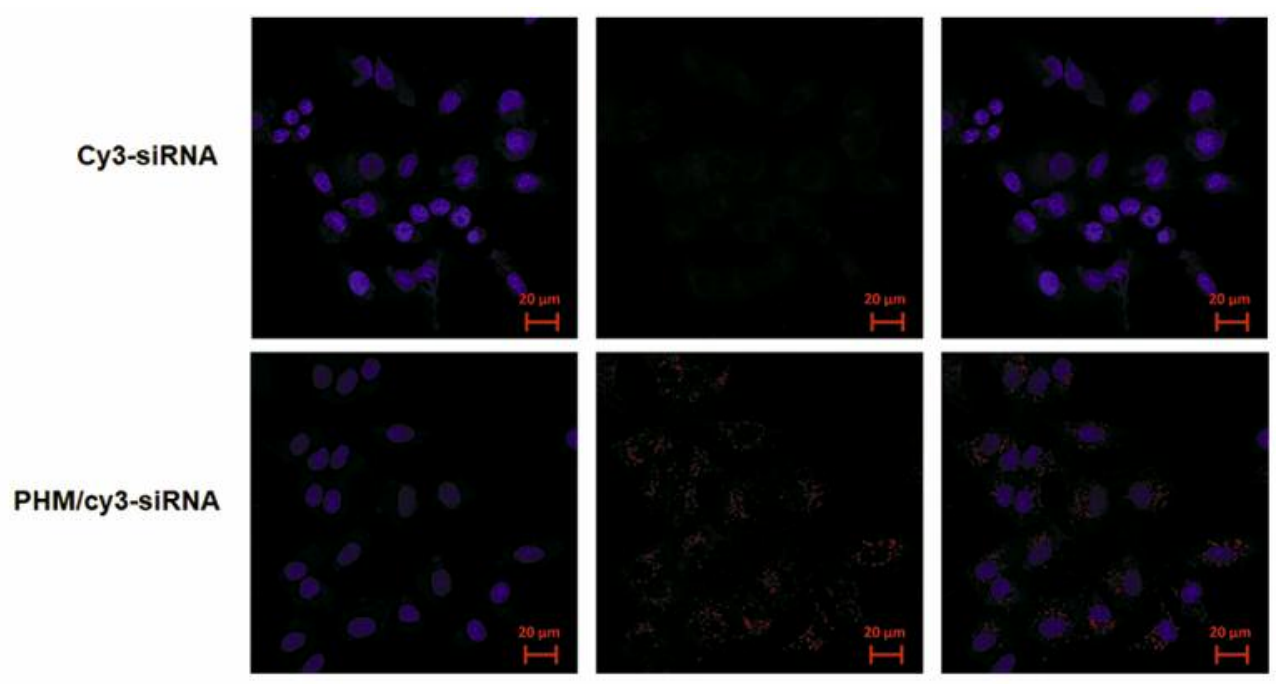

Figure 5. Cellular uptake of PHM/Cy3-siRNA by confocal laser scanning microscopy (CLSM). Naked Cy3-siRNA and PHM/ Cy3-siRNA treated A549 cells was visualized by CLSM at 4 h. The nucleus was stained blue by DAPI and siRNA is red. Red bar in the images was $20 \mu \mathrm{m}$.

The preparation and characterization of PHM. To investigate how the proportion of the cationic polymer of PEI-LA affected the properties of PHM, PHM was prepared by self-assembly of different weight ratios of PEI-LA to mPEG-LA. As the ratio of PEI-LA increased, the particle size of the PHM gradually increased from 86 to $220 \mathrm{~nm}$ (Figure 2A). However, the values of PDI decreased from 0.518 to 0.21 (Figure 2B). The PDI results indicated that the single amphiphilic polymer of mPEI-LA had a broad particle size distribution. As the proportion of the cationic polymer (PEI-LA) increased, the positive potential of the PHM also increased from $-0.75 \mathrm{mV}$ to $41 \mathrm{mV}$ (Figure $2 \mathrm{C}$ ). The charge of the PHM could be easily changed by adjusting the percentage of PEI-LA. However, the toxicity of PHM must be assessed. Upon intravenous injection, PHM may interact with red blood cells (RBCs). Thus, to assess the safety of PHM, the hemolytic activity was first investigated. As shown in Figure 2D, when the percentage was below 10, the PHM had relatively low hemolysis. When the weight $\%$ of PEI-LA was $2 \%$, the hemolytic activity was almost equivalent to mPEG-LA. Therefore, the PHM with $2 \%$ of PEI-LA was chosen as a safe candidate vector for siRNA delivery, and was used for all subsequent experiments.

Characterization of $\mathrm{PHM} / \mathrm{siRNA}$. Gel retardation assay was conducted to assess the ability of PHM to complex siRNA. As 


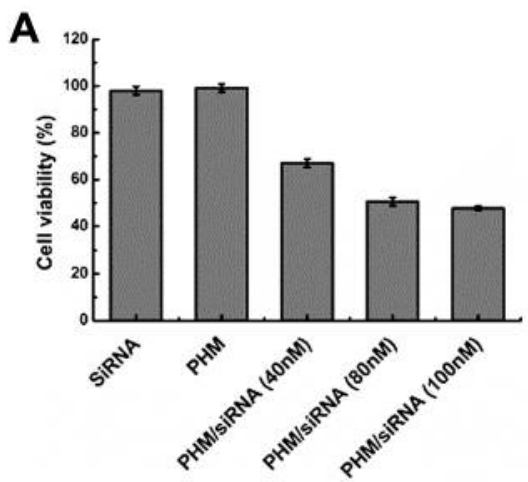

B

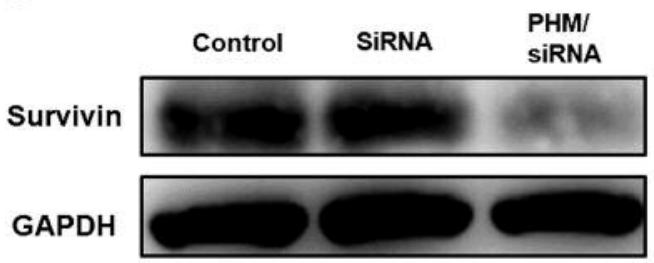

Figure 6. In vitro biological activities of PHM/survivin-siRNA in A549 cells. (A) The cytotoxicity of the PHM/survivin-siRNA with different concentrations of siRNA (40 nM, $80 \mathrm{nM}, 100 \mathrm{nM})$ was investigated after the formulations were incubated with cells for 48 $h(n=6$, mean $\pm S E M)$. (B) Protein expression of naked siRNA and PHM/survivin-siRNA treated cells was further measured by western blot assay.

shown in Figure 3A, PHM could successfully condense siRNA with an N/P ratio of $12 / 1$. The particle size, PDI, and zeta potential of the $\mathrm{PHM} / \mathrm{siRNA}(\mathrm{N} / \mathrm{P}=12 / 1)$ were $119.3 \pm 2.4 \mathrm{~nm}$, $0.245 \pm 0.017$ and $4.56 \pm 0.24 \mathrm{mV}(\mathrm{n}=3$, mean $\pm \mathrm{SEM})$, respectively. Compared to PHM, PHM/siRNA had a smaller particle size, lower zeta potential, and narrower particle size distribution, probably due to the electrostatic adsorption of siRNA and cationic polymer in PHM.

Cell uptake of PHM/Cy3-siRNA in A549 cells. Cellular uptake of PHM/Cy3-siRNA was investigated in A549 cells by flow cytometry and confocal laser-scanning microscopy (CLSM). Flow cytometry results showed that the PHM/Cy3-siRNAtreated cells had a higher mean fluorescence intensity compared to Cy3-siRNA treated cells (Figure 4A and B). Similar results were also obtained by CLSM. The red fluorescence intensity in the cytoplasm of cells treated with the PHM/Cy3-siRNA was stronger than that of the cells treated with the naked Cy3-siRNA (Figure 5). The results showed that PHM could efficiently deliver siRNA to the A549 cells.

In vitro biological activities of PHM/survivin-siRNA. Survivin has recently been found to be a crucial protein for tumor growth and metastasis and a promising therapeutic target (35). The inhibition of survivin expression may cause cell apoptosis or death and inhibit tumor growth (34). Survivin-siRNA was selected as a therapeutic agent for tumor therapy in this study. Cytotoxicity of PHM/survivin-siRNA was first studied in A549 cells by MTT assay. PHM/survivin-siRNA showed enhanced cell cytotoxicity compared to naked siRNA and PHM (Figure 6). PHM had no apparent cytotoxicity to the cells. As the concentration of siRNA loaded in PHM increased from 40 to $100 \mathrm{nM}$, the cell viability decreased from $67 \%$ to $47 \%$ (Figure 6). Simultaneously, the survivin protein expression in cells treated with PHM/survivin-siRNA (80 nM) was also greatly inhibited (Figure 6). These results indicated that PHM could facilitate transport of siRNA into the cells, reduce expression of targeted protein, and induce cell death.

Biodistribution of PHM/Cy5-siRNA in tumor-bearing mice. Efficient delivery of siRNA to the tumor site was a prerequisite for successful treatment. Therefore, we studied the biodistribution of PHM/Cy5-siRNA in tumor-bearing mice. As shown in Figure 7A, a fast clearance of free siRNA in tumorbearing mice was found. At the fourth $\mathrm{h}$, the red fluorescence signal in the tumor injected with Cy5-siRNA was barely visible. The same results were also reflected in the anatomical organs (Heart, Liver, Spleen, Lung, Kidney) and tumors at the sixth $\mathrm{h}$ after administration. The fluorescence signal of the free Cy5-siRNA injection mice was barely detectable in major organs and tumors other than the kidney (Figure 7A). However, PHM/Cy5-siRNA exhibited higher accumulation in solid tumors and lower fluorescence intensity in the kidney. This suggested that PHM could deliver Cy5-siRNA to the tumor site and protect Cy5-siRNA from fast clearance.

Antitumor efficacy of PHM/survivin-siRNA in tumor-bearing mice. Consistent with the in vitro results, tumor growth inhibition was significantly achieved in the PHM/survivinsiRNA treated group $\left({ }^{*} p<0.05\right)$ (Figure $8 \mathrm{~A}$ ). Body weight and histopathological analysis of major organs of the mice were measured to assess the systemic toxicity of the PHM/survivin-siRNA. As shown in Figure 8B, the body weight of the mice treated with $\mathrm{PHM}$ /survivin-siRNA increased steadily compared with the naked siRNA and saline group, indicating that the formulations had no significant systemic toxicity. Large areas of necrosis in tumor tissue were found in the PHM/siRNA treated group (indicated by the black arrow, Figure 9A), and the expression level of survivin protein was also greatly reduced (Figure 9B). There was no significant toxicity in the major organs of mice treated with $\mathrm{PHM} /$ survivin-siRNA compared to the 

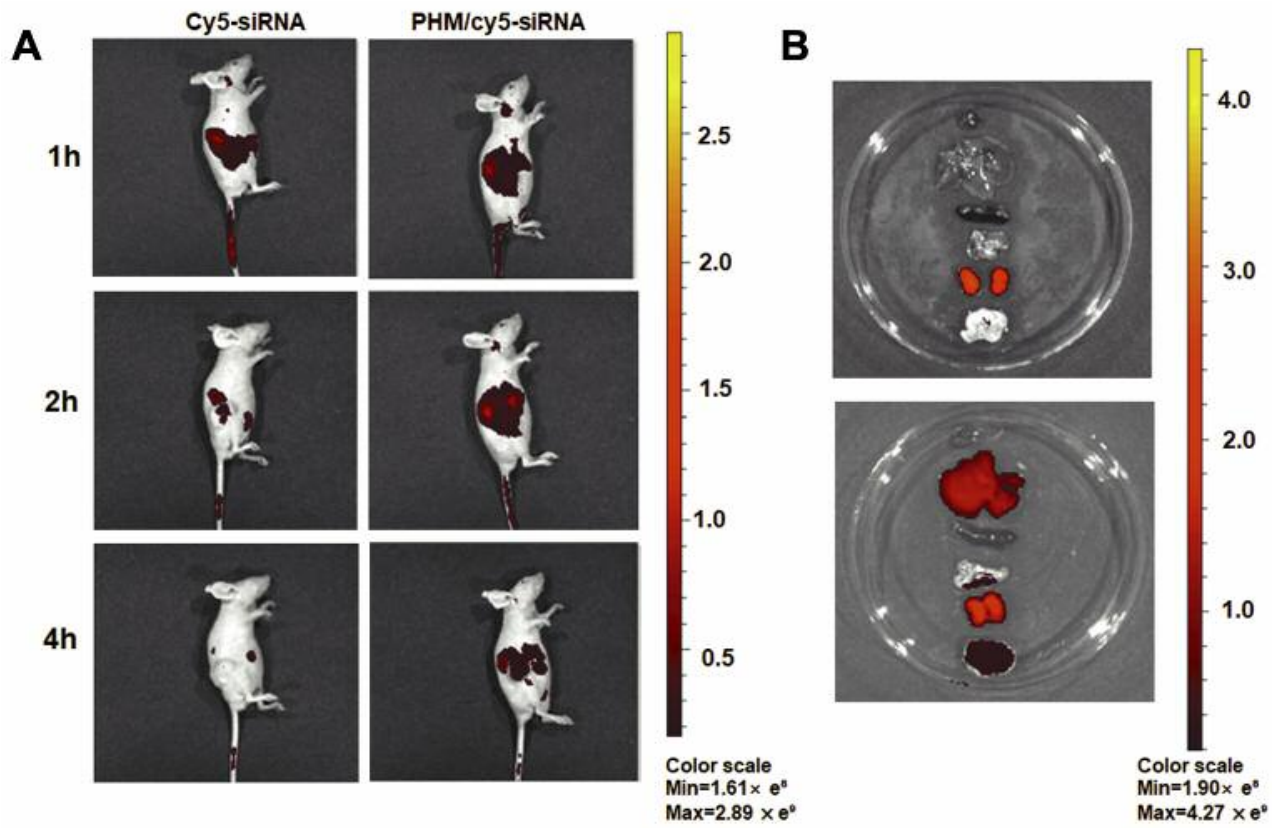

Figure 7. Bio-distribution of PHM/Cy5-siRNA in tumor-bearing mice. Real-time fluorescence imaging of tumor-bearing mice injected with naked Cy5-siRNA, PHM/Cy5-siRNA via tail vein at the first, second, and fourth hour after administration (Cy5-siRNA=0.8 nmol) (A). Ex vivo imaging of the tumors and organs excised from tumor-bearing mice at the sixth hour after injection $(B)$. Images were obtained by IVIS ${ }^{\circledR}$ in vivo imaging system with an optimized parameter (Excitation, $640 \mathrm{~nm}$; Emission, $680 \mathrm{~nm}$ ).
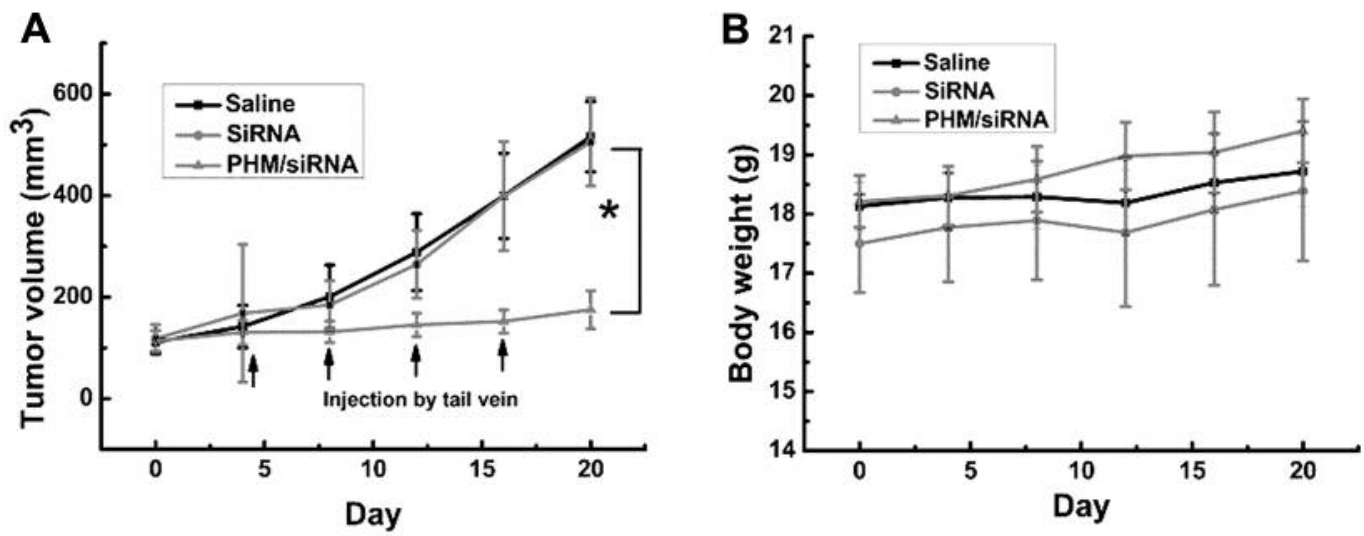

Figure 8. The therapeutic efficacy of PHM/siRNA in vivo. Tumor volume (A) and body weight (B) of tumor-bearing mice treated with saline, siRNA, or PHM/siRNA were measured every three days. $(n=5, * p<0.05$, PHM/siRNA compared to saline). Values are mean \pm SEM.

saline and siRNA group (Figure 10). PHM exhibited good biocompatibility and low toxicity as a non-viral delivery system for siRNA delivery.

\section{Discussion}

Effective delivery of siRNA to tumor cells is a prerequisite for its therapeutic effects. In our previous studies, we found that fatty acid-modified PEI, not only reduced the toxicity of
PEI, but also enhanced siRNA delivery efficiency (20-22). Although the PEI toxicity could be alleviated after hydrophobic modification (19), there are still many problems and obstacles for clinical application (14). PEG is widely used due to its good biocompatibility and long cycle time in the blood $(23,24,27)$. In this article, hydrophobic LA was successfully conjugated to PEI and mPEG-NH2 and two amphiphilic polymers (PEI-LA and mPEG-LA) were obtained. PHM was then prepared by self-assembly of PEI- 
A

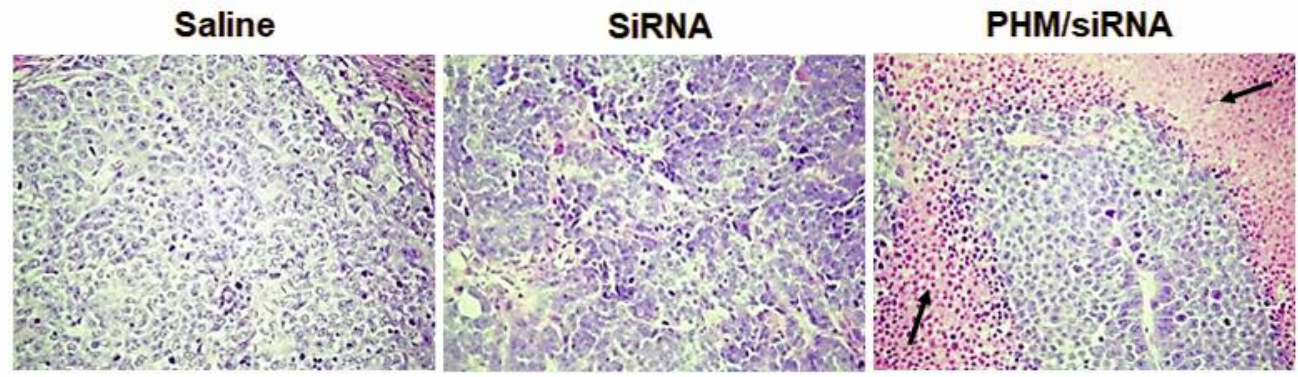

B

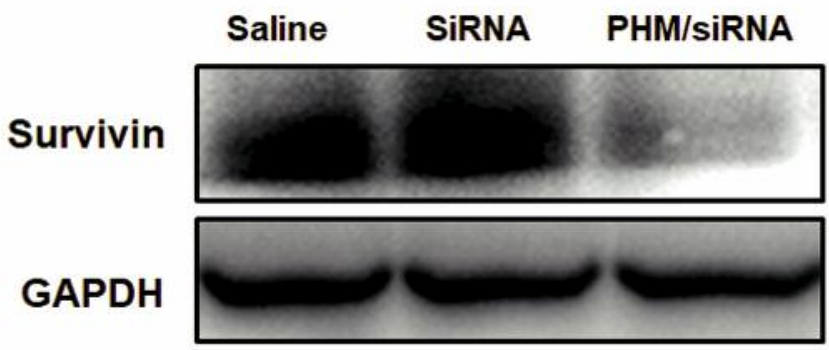

Figure 9. Histopathological analysis and protein expression of tumor tissues. Hematoxylin-eosin staining for tumor tissue necrosis (A) was investigated. Survivin expression in tumor tissues was determined by a western blot assay (B).

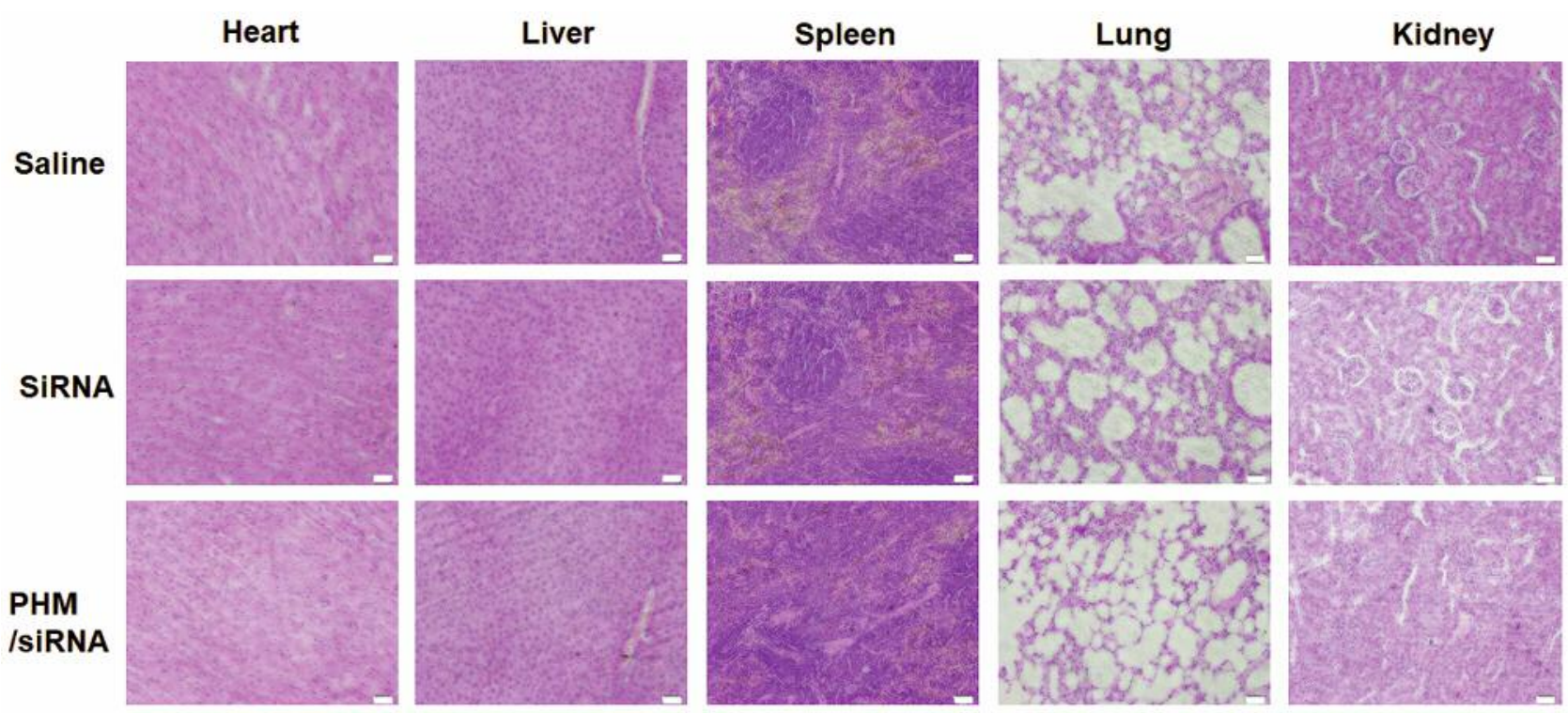

Figure 10. Toxicity analysis of major organs. Heart, spleen, lung, and kidney were dissected and stained with H\&E to assess the systemic toxicity of the drug formulations. The scale bar in the images was $20 \mu \mathrm{m}$.

LA and mPEG-LA to deliver siRNA (Figure 1). We first explored the property of different proportions of PEI-LA on the particle size, potential, and PDI of PHM. The advantage of PHM was that the charge of the PHM can be easily adjusted by changing the weight ratio of PEI-LA. In order to study the toxicity of PHM for intravenous injection in vivo, we also studied the hemolytic activity of PHM on RBCs.
When the weight ratio of PEI-LA was less than $10 \%$, the mixed micelles had good biocompatibility with RBCs (Figure 2 ). When the ratio of PEI-LA was $2 \%$, the hemolytic activity of PHM was almost the same as MPEG-LA. The results suggested that the addition of $\mathrm{MPEG}$ could reduce the toxicity of the PHM. Therefore, we prepared PHM with a 2\% weight ratio of PEI-LA to deliver siRNA. 
When the N/P ratio of PHM and siRNA was $12 / 1$, PHM could completely complex siRNA. PHM/siRNA complex had a narrow particle size (about $119 \mathrm{~nm}$ ) and a positive charge on particle size (about $4.56 \mathrm{mV}$ ) (Figure 3). Through flow cytometry and laser confocal microscopy experiments, we found that PHM can efficiently deliver siRNA into A549 cells (Figures 4 and 5). Simultaneously, cell toxicity and protein expression in vitro further demonstrated that siRNA delivered by PHM can reduce the expression of related proteins and inhibit the proliferation of tumor cells (Figure 6). The results indicated that PHM had successfully delivered siRNA into cells and achieved therapeutic efficacy in vitro. In vivo biodistribution studies further demonstrated that siRNA in PHM could be specifically gathered in tumor sites (Figure 7). The accumulation of siRNA at the tumor site may largely depend on the passive targeting ability of PHM achieved by enhanced permeability and retention effect (EPR) (36). Furthermore, the PHM/siRNA-administered mice exhibited significant inhibition of tumor growth compared to the saline and naked siRNA treated mice (Figure 8) in vivo. The histochemical staining and protein expression analysis of tumor tissues also showed large-area cell apoptosis and low expression of survivin protein in tumor tissues (Figure 9). Thus, PHM could efficiently deliver siRNA to tumor tissues, reduce related protein expression and finally inhibit tumor growth. Compared to active targeting delivery of carriers, passive targeting delivery of therapeutic agents, especially siRNA still plays an important role in the treatment of tumors without a target $(37,38)$. There are still no active targeting delivery systems for siRNA applied in the clinic (37). Further investigation of the PHM is, therefore, warranted.

\section{Conclusion}

In this study, an siRNA delivery system was developed based on PHM composed of two amphiphilic polymers. PHM/siRNA was effectively taken up by A549 cells and inhibited tumor cell growth by reducing tumor-related protein expression. Furthermore, studies in tumor-bearing mice showed that PHM/siRNA could enter into tumor tissues and inhibit tumor growth without systemic toxicity in vivo. PHM appears to be a promising and safe vehicle for siRNA delivery and may find utility in anticancer therapy.

\section{Conflicts of Interest}

The Authors declare no conflicts of interest.

\section{Authors' Contributions}

Conceptualization: L.T., J.X., R.J.L., and F.H.; experimental design: F.H., Y.S., C.Y., Z.L., Z.C., L.Z.; data analysis and validation: S.D., Z.C., Q.M., J.L.; F.H.; laboratory experimentation: F.H.; material acquisition: L.T.; manuscript preparation: original draft preparation, F.H.; review and editing: F.W., R.J.L. and F.H.; figure preparation: F.H.; supervision and project administration: L.T., Q.M, L.T., L.T., J.X. and J.L.

\section{Acknowledgements}

The biodistribution and histopathological analysis in this study were performed using the IVIS ${ }^{\circledR}$ spectrum system. Technical support was obtained from the Key Laboratory of Pathology and Biology Teaching of Ministry of Education and Basic Medical Experimental Teaching Center of Jilin University.

\section{References}

1 Chi X, Gatti $\mathrm{P}$ and Papoian T: Safety of antisense oligonucleotide and siRNA-based therapeutics. Drug Discov Today 22: 823-833, 2017. PMID: 28159625, DOI: 10.1016/ j.drudis.2017.01.013

2 Wilson RC and Doudna JA: Molecular mechanisms of RNA interference. Annu Rev Biophys 42: 217-239, 2013. PMID: 23654304, DOI:10.1146/annurev-biophys-083012-130404

3 Chakraborty C, Sharma AR, Sharma G, Doss CGP and Lee SS: Therapeutic miRNA and siRNA: Moving from bench to clinic as next generation medicine. Mol Ther Nucleic Acids 8: 132143, 2017. PMID: 28918016, DOI:10.1016/j.omtn.2017.06.005

4 Wittrup A and Lieberman J: Knocking down disease: a progress report on siRNA therapeutics. Nat Rev Genet 16: 543-552, 2015. PMID: 26281785, DOI: $10.1038 / \mathrm{nrg} 3978$

5 Kim HJ, Kim A, Miyata K and Kataoka K: Recent progress in development of siRNA delivery vehicles for cancer therapy. Adv Drug Deliv Rev 104: 61-77, 2016. PMID: 27352638, DOI:10.1016/ j.addr.2016.06.011

6 Kaczmarek JC, Kowalski PS and Anderson DG: Advances in the delivery of RNA therapeutics: from concept to clinical reality. Genome Med 9: 60, 2017. PMID: 28655327, DOI:10.1186/ s13073-017-0450-0

7 Chen $\mathrm{C}$, Yang $\mathrm{Z}$ and Tang X: Chemical modifications of nucleic acid drugs and their delivery systems for gene-based therapy. Med Res Rev 38: 829-869, 2018. PMID: 29315675, DOI: $10.1002 / \mathrm{med} .21479$

8 von Harpe A, Petersen H, Li Y and Kissel T: Characterization of commercially available and synthesized polyethylenimines for gene delivery. J Control Release 69: 309-322, 2000. PMID: 11064137.

9 Lai WF: In vivo nucleic acid delivery with PEI and its derivatives: Current status and perspectives. Expert Rev Med Devices 8: 173-185, 2011. PMID: 21381910, DOI:10.1586/ erd. 10.83

10 Ewe A, Hobel S, Heine C, Merz L, Kallendrusch S, Bechmann I, Merz F, Franke $\mathrm{H}$ and Aigner A: Optimized polyethylenimine (PEI)-based nanoparticles for siRNA delivery, analyzed in vitro and in an ex vivo tumor tissue slice culture model. Drug Deliv Transl Res 7: 206-216, 2017. PMID: 27334279, DOI:10.1007/ s13346-016-0306-y

11 Wang W, Balk M, Deng Z, Wischke C, Gossen M, Behl M, Ma $\mathrm{N}$ and Lendlein A: Engineering biodegradable micelles of polyethylenimine-based amphiphilic block copolymers for efficient DNA and siRNA delivery. J Control Release 242: 7179, 2016. PMID: 27498020, DOI:10.1016/j.jconrel.2016.08.004 
12 Zhang L, Lu Z, Zhao Q, Huang J, Shen H and Zhang Z: Enhanced chemotherapy efficacy by sequential delivery of siRNA and anticancer drugs using PEI-grafted graphene oxide. Small 7: 460-464, 2011. PMID: 21360803, DOI:10.1002/ smll.201001522

13 Zhou L, Xi Y, Chen M, Niu W, Wang M, Ma PX and Lei B: A highly antibacterial polymeric hybrid micelle with efficiently targeted anticancer siRNA delivery and anti-infection in vitro/in vivo. Nanoscale 10: 17304-17317, 2018. PMID: 30198034, DOI: $10.1039 / \mathrm{c} 8 \mathrm{nr} 03001 \mathrm{~d}$

14 Zhou Z, Liu X, Zhu D, Wang Y, Zhang Z, Zhou X, Qiu N, Chen $X$ and Shen $Y$ : Nonviral cancer gene therapy: Delivery cascade and vector nanoproperty integration. Adv Drug Deliv Rev 115: 115154, 2017. PMID: 28778715, DOI:10.1016/j.addr.2017. 07.021

15 Zintchenko A, Philipp A, Dehshahri A and Wagner E: Simple modifications of branched PEI lead to highly efficient siRNA carriers with low toxicity. Bioconjug Chem 19: 1448-1455, 2008. PMID: 18553894, DOI:10.1021/bc800065f

16 Zhang QY, Ho PY, Tu MJ, Jilek JL, Chen QX, Zeng S and Yu AM: Lipidation of polyethylenimine-based polyplex increases serum stability of bioengineered RNAi agents and offers more consistent tumoral gene knockdown in vivo. Int J Pharm 547: 537544, 2018. PMID: 29894758, DOI:10.1016/j.ijpharm.2018.06.026

$17 \mathrm{Wu}$ D, Zhang Y, Xu X, Guo T, Xie D, Zhu R, Chen S, Ramakrishna S and He L: RGD/TAT-functionalized chitosan-graftPEI-PEG gene nanovector for sustained delivery of NT-3 for potential application in neural regeneration. Acta Biomater 72: 266277, 2018. PMID: 29578088, DOI:10.1016/j.actbio.2018.03.030

18 Yin Y, Lee J, Kim N, Lee J, Lim S, Kim E, Park J, Lee M and Jeong J: Inhibition of tumor growth via systemic siRNA delivery using reducible bile acid-conjugated polyethylenimine. Polymers 10: 953, 2018. DOI:10.3390/polym10090953

19 Dunn AW, Kalinichenko VV and Shi D: Highly efficient in vivo targeting of the pulmonary endothelium using novel modifications of polyethylenimine: an importance of charge. Adv Healthc Mater 7: e1800876, 2018. PMID: 30398703, DOI:10.1002/ adhm.2018 00876

20 Xie J, Teng L, Yang Z, Zhou C, Liu Y, Yung BC and Lee RJ: A polyethylenimine-linoleic acid conjugate for antisense oligonucleotide delivery. Biomed Res Int 2013: 710502, 2013. PMID: 23862153, DOI:10.1155/2013/710502

21 Teng LS, Xie J, Teng LR and Lee RJ: Enhanced siRNA delivery using oleic acid derivative of polyethylenimine. Anticancer Res 32: 1267-1271, 2012. PMID: 22493358.

22 Alshamsan A, Haddadi A, Incani V, Samuel J, Lavasanifar A and Uludag H: Formulation and delivery of siRNA by oleic acid and stearic acid modified polyethylenimine. Mol Pharm 6: 121-133, 2009. PMID: 19053537, DOI:10.1021/mp8000815

23 Knop K, Hoogenboom R, Fischer D and Schubert US: Poly(ethylene glycol) in drug delivery: pros and cons as well as potential alternatives. Angew Chem Int Ed Engl 49: 6288-6308, 2010. PMID: 20648499, DOI:10.1002/anie.200902672

24 Kolate A, Baradia D, Patil S, Vhora I, Kore G and Misra A: PEG a versatile conjugating ligand for drugs and drug delivery systems. J Control Release 192: 67-81, 2014. PMID: 24997275, DOI: 10.1016/j.jconrel.2014.06.046

25 Rabanel JM, Hildgen P and Banquy X: Assessment of PEG on polymeric particles surface, a key step in drug carrier translation. J Control Release 185: 71-87, 2014. PMID: 24768790, DOI: 10.1016/j.jconrel.2014.04.017
26 Bhang SH, Kim K, Rhee WJ and Shim MS: Bioreducible polyspermine-based gene carriers for efficient siRNA delivery: effects of peg conjugation on gene silencing efficiency. Macromolecular Res 26: 1135-1142, 2018. DOI:10.1007/s13233019-7027-2

27 Sant S, Poulin S and Hildgen P: Effect of polymer architecture on surface properties, plasma protein adsorption, and cellular interactions of pegylated nanoparticles. J Biomed Mater Res A 87: 885-895, 2008. PMID: 18228249, DOI:10.1002/jbm.a.31800

28 Arami S, Mahdavi M, Rashidi MR, Yekta R, Rahnamay M, Molavi L, Hejazi MS and Samadi N: Apoptosis induction activity and molecular docking studies of survivin siRNA carried by Fe3O4PEG-LAC-chitosan-PEI nanoparticles in MCF-7 human breast cancer cells. J Pharm Biomed Anal 142: 145-154, 2017. PMID: 28505590, DOI:10.1016/j.jpba.2017.04.025

29 Cagel M, Tesan FC, Bernabeu E, Salgueiro MJ, Zubillaga MB, Moretton MA and Chiappetta DA: Polymeric mixed micelles as nanomedicines: Achievements and perspectives. Eur J Pharm Biopharm 113: 211-228, 2017. PMID: 28087380, DOI:10.1016/ j.ejpb.2016.12.019

30 Yoo HS and Park TG: Folate receptor targeted biodegradable polymeric doxorubicin micelles. J Control Release 96: 273-283, 2004. PMID: 15081218, DOI:10.1016/j.jconrel.2004.02.003

31 Wang Q, Jiang H, Li Y, Chen W, Li H, Peng K, Zhang Z and Sun $\mathrm{X}$ : Targeting NF-kB signaling with polymeric hybrid micelles that co-deliver siRNA and dexamethasone for arthritis therapy. Biomaterials 122: 10-22, 2017. PMID: 28107661, DOI:10.1016/ j.biomaterials.2017.01.008

32 Feng L, Yang X, Shi X, Tan X, Peng R, Wang J and Liu Z: Polyethylene glycol and polyethylenimine dual-functionalized nanographene oxide for photothermally enhanced gene delivery. Small 9: 1989-1997, 2013. PMID: 23292791, DOI:10.1002/ smll.201202538

33 Yang L, Wu X, Liu F, Duan Y and Li S: Novel biodegradable polylactide/poly(ethylene glycol) micelles prepared by direct dissolution method for controlled delivery of anticancer drugs. Pharm Res 26: 2332-2342, 2009. PMID: 19669098, DOI:10.1007/ s11095-009-9949-4

34 Peery RC, Liu JY and Zhang JT: Targeting survivin for therapeutic discovery: past, present, and future promises. Drug Discov Today 22: 1466-1477, 2017. PMID: 28577912, DOI:10.1016/j.drudis. 2017.05.009

35 Ryan BM, O’Donovan N and Duffy MJ: Survivin: a new target for anti-cancer therapy. Cancer Treat Rev 35: 553-562, 2009. PMID: 19559538, DOI:10.1016/j.ctrv.2009.05.003

36 Perry JL, Reuter KG, Luft JC, Pecot CV, Zamboni W and DeSimone JM: Mediating Passive Tumor Accumulation through Particle Size, Tumor Type, and Location. Nano Lett 17: 2879-2886, 2017. PMID: 28287740, DOI:10.1021/acs.nanolett.7b00021

37 Park DH, Cho J, Kwon OJ, Yun CO and Choy JH: Biodegradable inorganic nanovector: passive versus active tumor targeting in siRNA transportation. Angew Chem Int Ed Engl 55: 4582-4586, 2016. PMID: 26879376, DOI:10.1002/anie.201510844

38 Li R, Zheng K, Yuan C, Chen Z and Huang M: Be active or not: the relative contribution of active and passive tumor targeting of nanomaterials. Nanotheranostics 1: 346-357, 2017. PMID: 2907 1198, DOI:10.7150/ntno. 19380

Received February 2, 2019

Revised February 17, 2019

Accepted February 19, 2019 